\title{
COVID-19 aus der Perspektive der Palliativmedizin und Hospizbetreuung
}

Gerold Muhri und Désirée Amschl-Strablegg, befragt von Johann Platzer

Das Krankenhaus der Elisabethinen in Graz ist ein gemeinnütziges Akutkrankenhaus mit rund 180 Betten auf insgesamt neun Stationen. Jährlich werden rund 33.500 PatientInnen ambulant und stationär versorgt. Eigentümer und Träger des Krankenhauses ist der Konvent der Elisabethinen. Durch die Spitalskooperation der Elisabethinen mit den Barmherzigen Brüdern (Ordenskrankenhaus Graz-Mitte) und bauliche Adaptionsmaßnahmen werden bis zum Jahr 2025 rund 220 Betten zur Verfügung stehen. Dabei soll der Fokus vor allem auf eine umfassende Altersversorgung und Altersmedizin gelegt werden. Ergänzend zu der seit 1998 bestehenden Palliativstation mit insgesamt acht Betten gibt es bei den Elisabethinen auch zwei Hospize mit je zwei Betten, und zwar das hauseigene Hospiz St. Elisabeth (seit 2018) sowie das VinziDorf-Hospiz (seit 2017). Dieses ist Europas erstes Hospiz für obdachlose Menschen, in welchem unheilbar kranke Menschen bis zu ihrem Lebensende umfassend betreut werden.

Auf der Palliativstation werden PatientInnen mit chronischen und fortgeschrittenen Erkrankungen betreut, die aufgrund der Schwere der Beschwerden nicht mehr zu Hause oder in anderen Einrichtungen behandelt werden können. Ziel ist es, durch die Behandlung von akuten Symptomen die Lebensqualität der PatientInnen so zu verbessern, dass diese wieder in häusliche Pflege oder in eine Pflegeeinrichtung entlassen werden können. Darüber hinaus berät ein Palliativkonsiliarsdienst in komplexen palliativen Fragestellungen. Dieses mulitprofessionelle Team, bestehend aus Sozialarbeit, Pflege und Medizin, führt ausführliche Vor-Ort-Gespräche mit dem Behandlungsteam, PatientInnen und Angehörigen auf anderen Stationen und in anderen Kooperationskrankenhäusern.

Oberarzt Dr. Gerold Muhri ist medizinischer Leiter des Bereichs Palliativmedizin und Hospizbetreuung. Sein Team besteht aus vier weiteren Ärztinnen und Ärzten. Die diplomierte psychiatrische Gesundheits- und Krankenpflegerin Désirée Amschl-Strablegg hat die Bereichsleitung für Hospiz und Palliativ inne und ist seit 2012 als Pflegedienstleiterin für insgesamt 22 Pflegende verantwortlich. COVID-19 hat besonders den Gesundheits- und Pflegebereich mit neuen, zuvor kaum geahnten Herausfor- 
derungen konfrontiert. In einem Interview mit Johann Platzer schildern die beiden ihre Erfahrungen während der Corona-Pandemie aus der Perspektive der Palliativmedizin und Hospizbetreuung.

Platzer: Ich möchte zunächst mit ein paar allgemeinen Fragen beginnen: Glauben Sie, dass die Corona-Pandemie unser Denken über Sterben und Tod verändert hat?

Amschl-Strablegg: Ja, aber nicht nachhaltig. Sobald wir in unseren Alltag zurückkehren, glaube ich, dass das Sterben auch wieder in den Hintergrund treten wird.

Mubri: Ich glaube auch, dass der ,Rechtsanspruch' unserer Gesellschaft, das Leben bis ins hohe Alter haben zu dürfen, verschwunden ist - vor allem während des ersten Lockdowns. Während dieser Zeit ist das Sterben und der Tod präsenter geworden - mit all den Ängsten und Tabuthemen; auch bei Menschen, bei denen Krankheit und Tod nicht im Fokus waren. Deshalb waren auch viel Solidarität und Betroffenheit spürbar, aber ich nehme jetzt schon wieder ein Verschwinden dieser Solidarität wahr. Auch das Bewusstsein, dass es dieses Recht auf Gesundheit nicht gibt, das schwindet.

\section{Platzer: Nehmen Sie derzeit Veränderungen in unserer Sterbekultur wabr?}

Mubri: Das glaube ich nicht, zumindest nicht durch Corona.

Amschl-Strablegg: Eine Veränderung der Sterbekultur durch Corona nehme ich auch nicht wahr. Ich glaube jedoch, dass in den vergangenen Jahren der Autonomiewunsch immer größer geworden ist. Dabei meine ich nicht nur das momentan aktuelle Thema assistierter Suizid. Unabhängig davon nehme ich in der PatientInnenbetreuung trotzdem verstärkt dieses Autonomiebedürfnis wahr. Das hat sich in den vergangenen Jahren schon verändert.

Mubri: Ich glaube, die Sterbekultur insgesamt liegt schon auch einem Wandel zugrunde. Zum einen am Land: die Natürlichkeit des Todes und des Sterbens, die wir von unseren Großeltern gewohnt waren, diese gibt es nach wie vor. Das habe ich am Land nach wie vor erlebt. In der Stadt waren vor fünf bis zehn Jahren die Themen Krankheit, Tod und Sterben große Tabu-Themen. Wenn ich heute im Stadtkrankenhaus das Sterben anspreche und dann noch eine Palliative Care anspreche, sind das Herz und die Ohren diesem Thema eher zugewandt als sie es noch vor etwa zehn Jahren waren. 
Amschl-Strablegg: Trotzdem ist die Institutionalisierung des Sterbens nach wie vor präsent. Wir sind noch nicht so weit, dass wir das Sterben auch zu Hause wieder zulassen wollen.

\section{„Im Bereich unserer Palliative Care sind die stabilisierenden Strukturen noch einmal klarer und pointierter herausgekommen"}

Platzer: Krisen, so sagt man mitunter, machen vorhandene Strukturen sichtbar. Welche Strukturen sind bei Ibrer Arbeit in der Palliativmedizin und Hospizbetreuung sichtbar geworden?

Mubri: Im Bereich unserer Palliative Care sind die stabilisierenden Strukturen noch einmal klarer und pointierter herausgekommen. Wir haben, glaube ich, im gesamten Team einen hohen Anspruch, Verantwortung zu übernehmen und Menschen in ihrer Krise gut zu begleiten und Angehörige gut zu stützen. Damit sind wir auch in der Corona-Zeit gut zurechtgekommen. Wir haben uns bemüht, in den Strukturen gut drinnen zu bleiben und uns daran festzuhalten; und wir haben uns bemüht, die Angst, die bei allen MitarbeiterInnen, bei allen PatientInnen und Angehörigen da war, wahrzunehmen und darauf einzugehen. So haben wir zum Beispiel eine Corona-Kerze während dieser Zeit etabliert, die wir bei unseren Besprechungen angezündet haben - manchmal auch mit einem ausgesprochenen Gebet von „Danke und Bitte“; also „Danke“, dass bei uns gerade niemand an Corona erkrankt ist und „Bitte“ für all jene, die von Corona direkt betroffen sind und daran leiden. Die Ängste und das Leiden, die bei Einzelnen und in der Gesellschaft da waren, haben wir also nicht ausgeklammert. Wir haben uns an den bestehenden Strukturen festgehalten, um im Alltag unsere PatientInnen gut begleiten zu können.

Amschl-Strablegg: Ja, wir waren vieles ja schon vorher gewohnt, zum Beispiel was die Hygiene betrifft. Wenn wir PatientInnen mit einem Keim behandeln, dann mussten wir ja immer schon gewisse Schutzmaßnahmen treffen. Das ist uns ja nicht fremd, und diese Schutzmaßnahmen mussten wir dann eben den ganzen Tag über anwenden. Es ist für mich rückblickend äußerst überraschend, wie wenig positive Corona-Fälle wir in unserem Team hatten. Es sind kaum MitarbeiterInnen an Corona erkrankt und auch auf der Station hat es, mit Ausnahme eines einziges ,Falles', nichts gegeben. Ich finde es sehr bemerkenswert, dass unsere Strukturen und unser Verantwortungsgefühl hier uns gut durch diese Zeit durchgetragen haben. Es muss also ein relativ hohes Level an Verantwortungsgefühl schon immer da gewesen sein, denn es hat nicht gestresst, plötzlich noch 
konsequenter zu sein. Die Veränderungen außerhalb der Station waren meines Erachtens weit größer als innerhalb der Station.

Platzer: Wie sieht eigentlich Ihr multiprofessionelles Team auf der Palliativstation bzw. im Palliativkonsiliarsdienst konkret aus?

Muhri: Bei uns gibt es insgesamt sechs Berufsgruppen, die immer sehr eng miteinander zusammenarbeiten: am engsten sicherlich die Pflege, die Medizin und die Sozialarbeit; es folgen die Physiotherapie, die Psychotherapie und die Seelsorge, und als Ehrenamtliche haben wir noch HospizbegleiterInnen und eine Therapeutin für die tiergestützte Therapie in unserer Einheit. Wir haben da eine flache Hierarchie und versuchen, achtsam auf Augenhöhe miteinander umzugehen.

Platzer: Hospiz und Palliative Care umfassen ja die ganzheitliche Betreuung und Begleitung von Menschen mit schweren Erkrankungen, wenn eine Heilung meist nicht mehr möglich ist. Hat sich Ihres Erachtens die Wahrnehmung über Hospiz und Palliative Care während der Pandemie verändert?

Muhri: Gesellschaftlich eher nicht, aber in unserem Krankenhaus sehr wohl, insofern wir uns bemüht haben, die Covid-Stationen durch unsere Erfahrungen mit Sterben und Tod mitzubegleiten und mitzutragen. Das betraf bzw. betrifft sowohl die akut Erkrankten und die Angehörigen, besonders aber auch das Behandlungsteam einer normalen Station. Und ich glaube schon, dass da eine gewisse Steigerung der Wertschätzung von Hospiz und Palliative Care spürbar geworden ist.

Amschl-Strablegg: Ja, denn wir sind ja im Gegensatz zu anderen Stationen ständig mit dem Sterben konfrontiert. Kolleginnen und Kollegen haben mir erzählt, dass der Allgemeinzustand vieler ihrer PatientInnen zu Beginn ja meist sehr gut war. Viele waren auf dem Weg der Besserung. Plötzlich hat sich dann aber deren Allgemeinzustand durch eine Covid-Erkrankung verschlechtert und diese sind dann relativ rasch verstorben; und diese plötzlichen Wendungen in den Krankheitsverläufen waren für viele KollegInnen sehr belastend.

Platzer: Hat es viele solche Verläufe gegeben?

Mubri: Ja, im Krankenhaus sind schon sehr viele an Corona verstorben. Oft sind Menschen mit einem leichten Infekt von zu Hause mit dem ,Seuchen-Taxi' abgeholt worden und dann aber nicht mehr nach Hause zurückgekommen. Das war für jeden Einzelnen in der Familie eine Tragödie. Aber auch die KollegInnen auf der Intensivstation haben bei uns einen 
hohen Leidensdruck gehabt. Diese haben die PatientInnen mit all ihren Bemühungen intensivmedizinisch versorgt und behandelt; gleichzeitig haben sie im Vorfeld schon oft gewusst, dass die PatientInnen mit hoher Wahrscheinlichkeit nicht überleben werden.

Amschl-Strablegg: Erst jetzt, wo sich die Situation etwas entspannt, merkt man, was dies alles mit dem Intensivteam gemacht hat. So kommt z. B. vom Intensivpflegeteam vermehrt der Wunsch nach Supervision auf.

„Mir ist aufgefallen, dass ein Großteil der Betroffenen es gar nicht wollte, als ,vulnerabel' wahrgenommen zu werden"

Platzer: Ältere und schwer kranke Menschen sind vor allem zu Beginn der Corona-Pandemie vor allem als, Risikogruppe $e^{‘}$ und als, zu Beschützende und Schwache' wahrgenommen worden. Welche Auswirkungen hatte diese Fokussierung Ihres Erachtens auf die Betroffenen?

Amschl-Strablegg: Mir ist aufgefallen, dass ein Großteil der Betroffenen es gar nicht wollte, als vulnerabel wahrgenommen zu werden. Auf diese Gruppe haben wir meines Erachtens viel zu sehr den Fokus gelegt und dabei andere Gruppen, wie zum Beispiel Kinder, vergessen - zumindest gesellschaftlich.

Mubri: Ich glaube, dass es gut ist, dass unsere Achtung und Sorge auf vorerkrankte Menschen gelegt wurde bzw. wird. Die Konsequenz, dass wir diese zu beschützenden Menschen mitunter, weggesperrt' haben und normalen Kontakt unterbunden haben, hat aber weder das Leben, noch die Lebensqualität dieser Menschen gefördert.

Platzer: Schauen wir vielleicht noch einmal auf die vergangenen Monate etwas genauer zurück: Inwiefern haben sich dabei die berufspezifischen Rollen verändert, und welche Konturen einzelner Disziplinen sind dabei besonders sichtbar geworden?

Amschl-Strablegg: Die Pflege ist durch Corona - zusammen mit der Medizin - auf jeden Fall ganz stark in den Fokus gerückt. Ich finde auch, dass die Rolle der Seelsorge sehr in den Vordergrund getreten ist. Mich hat aber auch etwas irritiert, dass gewisse Berufsgruppen auf einmal weniger präsent waren. So hat z. B. die Sozialarbeit plötzlich im Homeoffice gearbeitet. Das war für mich nicht ganz nachvollziehbar.

Mubri: Ich glaube, dass sich bei uns die Konturen insofern geschärft haben, dass wir erkannt haben, dass dort, wo Hilfe notwendig ist, wir 
diese auch leisten. Wir haben bewusst das Risiko auf uns genommen, einen positiv getesteten Corona-Patienten zu betreuen, weil wir diesem Menschen unsere Unterstützung einfach schuldig waren. Ich bin auch sehr froh und echt stolz, dass alle in unserem Team bereit waren, zu positiv getesteten Corona-Patienten hinzugehen und Hilfe zu leisten. Das inkludiert alle Berufsgruppen bei uns; erwähnen möchte ich aber insbesondere die Seelsorge. Ich habe nämlich mit Traurigkeit beäugt, dass in anderen Einrichtungen gerade die Seelsorge in einer für Seele und Körper sehr bedrängenden Zeit nicht da sein durfte oder wollte oder konnte bzw. da war.

Amschl-Strablegg: Das ist aber natürlich auch etwas nachvollziehbar, da man am Anfang der Pandemie einfach viel zu wenig gewusst hat und auch Schutzausrüstung nicht immer vorhanden war. Bei den älteren ehrenamtlichen SeelsorgerInnen, aber auch bei HospizbegleiterInnen gingen die Meinungen diesbezüglich zwar auseinander, aber bei uns hatten alle Meinungen ihren Platz: Manche wollten einfach zu Hause bleiben, manche hingegen wollten unbedingt PatientInnen besuchen. Es hat beide Extreme gegeben, und alles hatte ihren Platz. In unserem Haus habe ich diesbezüglich diese Wertfreiheit und Offenheit als sehr positiv wahrgenommen.

\section{„Die Rolle der Seelsorge muss innerhalb der Gesundheitsberufe viel mehr bonoriert werden"}

Platzer: Glauben Sie, dass es auch nachhaltige Auswirkungen auf einzelne Berufsgruppen geben wird?

Amschl-Strablegg: Ja, vor allem auf die Pflege. Ein Pflegemangel droht nicht nur, ein solcher ist vielmehr schon längst da. Es fürchten sich auch alle vor dem Pflexit, also davor, dass viele den Pflegeberuf aufgrund der erfahrenen Überbelastung verlassen werden. Das wird so auch passieren. Jene, die es sich finanziell leisten können, werden wahrscheinlich den Beruf wechseln. Man könnte die Situation der Pflege so zusammenfassen: $\mathrm{Zu}$ wenig Geld, zu wenig Freizeit, zu hohe Belastung und zu wenig Anerkennung. Was ich mir auch wünschen würde, ist, dass die Aufwertung der Seelsorge von Dauer ist. Es muss vielmehr gesehen werden, dass die Seelsorge in dieser Zeit da war. Es wird ja z. B. in den Niederlanden schon diskutiert, die Seelsorge zugunsten der Psychologie oder Psychotherapie aus den Krankenhäusern zu entfernen. Das muss unbedingt vermieden werden. 
Mubri: Auf jeden Fall! Die Seelsorge kann niemals durch einen Psychologen ersetzt werden. Da hilft es auch nichts, wenn man diesen mit einem ,Spritual-Care-Stempel'versieht. Das halte ich für grundfalsch! Die Rolle der Seelsorge muss innerhalb der Gesundheitsberufe viel mehr honoriert werden.

Amschl-Strablegg: Und die SeelsorgerInnen könnten und sollten auch etwas selbstbewusster auftreten.

Mubri: Das stimmt. Das Selbstbewusstsein der Seelsorge ist auf jeden Fall ausbaufähig. In vielen Spitälern habe ich erlebt, dass die Krankenhausseelsorge oft nur schüchtern um die Ecke schaut und auch nicht ihren Platz zugewiesen bekommt. Ich bin unheimlich dankbar, dass wir in unserem Haus eine sehr präsente Krankenhausseelsorge haben. Wir bemühen uns auch, ihr wirklich einen gleichrangigen Platz an unserem Tisch zu geben, weil sie in der Versorgung eines Palliativpatienten oft eine sehr wichtige Rolle einnimmt, manchmal sogar die wichtigste!

Amschl-Strablegg: Ja, das sehe ich auch so.

Platzer: Welche Konzepte und Leitlinien haben sich bei Ihrer Arbeit während der Pandemie bewährt?

Amschl-Strablegg: Zu Beginn der Pandemie habe ich es schon irrsinnig bewundert, mit welcher Sicherheit wir durch diese geführt worden sind, obwohl man so wenig darüber gewusst hat. Aus der kollegialen Führung hat sich ein Krisenstab gebildet, und da stand natürlich die KrankenhausHygiene zunächst im Mittelpunkt. Die Hygiene zählt ja im Allgemeinen nicht $\mathrm{zu}$ den beliebtesten Abteilungen eines Krankenhauses. Sie muss auch immer sehr strukturiert arbeiten. Plötzlich war diese dann aber an vorderster Front, und ihre Strukturiertheit hat sich irrsinnig bewährt. Wir hatten auch stets die für uns relevanten Informationen, es gab immer wieder Handlungsanweisungen über Hygienemaßnahmen. Auch die Krankenhaus-Apotheke hat sich extrem gut bewährt, sowohl was die Beschaffung, als auch die Herstellung von Desinfektionsmitteln betrifft.

Muhri: Die Hygienemaßnahmen haben sich tatsächlich sehr gut bewährt. Ich bin auch sehr dankbar darüber, dass wir mit viel Verantwortungsbewusstsein und Fingerspitzengefühl die Vorgaben und Leitlinien umsetzen haben können und trotzdem eine gewisse Berührung mit den Menschen, die in Not waren, möglich machen haben können.

Amschl-Strablegg: Was mir diesbezüglich dazu noch einfällt: Zunächst wurde ja kaum jemand in die Krankenhäuser hineingelassen. Wir haben uns dann aber relativ rasch darum bemüht, dass die Ehrenamtlichen wieder zu uns kommen können - natürlich nur jene, die es auch wollten. 
Das heißt: Wir haben am Konzept der multiprofessionellen Begleitung festgehalten, und das hat sich rückblickend auch sehr bewährt. Alle haben sich an die Maßnahmen gehalten, und wir hatten sogar wieder HospizpraktikantInnen bei uns. Da gab es eben auch Rückendeckung im Haus.

Mubri: Ja, ich finde es sehr schön, dass unsere Hausleitung uns den Freiraum gegeben hat, dass wir jederzeit Angehörigenbesuch möglich machen konnten, wenn es notwendig war - und das ist im palliativen Segment eben sehr oft, wenn nicht immer der Fall. In all unseren Einrichtungen war immer Besuch möglich.

\section{„Die Krise gehört zu unserem Alltag“}

Platzer: Die Corona-Krise war und ist für alle Beteiligten eine hoch emotionale Zeit. Wie hat sich dies bei Ibren Mitarbeiterinnen und Mitarbeitern bemerkbar gemacht?

Mubri: Immer dann, wenn bei uns im Team etwas emotional belastend wird, steigt der Redebedarf. So hat auch Corona den Redebedarf und die Emotionen gesteigert bzw. verändert. Ich glaube, dass es uns gut gelungen ist, die Maßnahmen so umzusetzen, dass sie allen MitarbeiterInnen Sicherheit gegeben haben und dass dabei trotzdem palliative Arbeit in einem sinnhaften Tun weiterhin möglich war. Das ging aber nur durch ständiges Durchbesprechen der jeweiligen Situation: Was braucht es nun an Vernunft bei der Umsetzung von Maßnahmen? Und was braucht es gleichzeitig für eine palliativberührende Versorgung?

Platzer: Welche ,Räume' gibt es diesbezüglich auf Ihrer Station, damit dieser angesprochene Redebedarf gestillt werden konnte bzw. kann?

Mubri: Reden gehört bei uns zur Alltagskultur. Bei uns ist fast jeder Raum gut und geeignet, um in Resonanz zu gehen. Das wird auch genützt. Wir haben natürlich auch strukturierte Besprechungen. Die Tagesplanung zum Beispiel findet bei uns täglich in der Früh statt, und wir haben auch die interdisziplinäre PatientInnenbesprechung, wo alle Berufsgruppen zusammenkommen. Das sind die fixen Besprechungs-Zeiten, wo natürlich auch Corona und seine Ängste ihren Raum hatten. Es hat aber auch bei vielen Visiten oder sonstigen Begegnungen in der Teeküche und am Gang natürlich unterstützende Gespräche gegeben, und wir nutzten und nutzen auf unserer Station auch immer wieder Einzel- und Gruppensupervisionen, um ins Gespräch zu gehen. 
Amschl-Strablegg: Das ist aber nicht überall so in unserem Haus. Der Redebedarf ist überall gestiegen, die Möglichkeiten zum Reden waren aber oft begrenzt. Da gibt es zwischen dem Palliativbereich und anderen Abteilungen gravierende Unterschiede. Ich habe mit vielen KollegInnen im Haus gesprochen. Für viele war es wirklich katastrophal, sich nicht mehr untereinander austauschen zu können, weil man ja ständig Schutzausrüstung tragen musste. Die Sozialräume sind ja oft sehr klein. Es hat auch kein gemeinsames Essen mehr gegeben. Das heißt, das so genannte Informelle ist komplett weggefallen. Vor allem die Pflege betreibt sehr viel Psychohygiene informell, z. B. beim Kaffeetrinken oder beim Essen. Ich weiß von vielen Hauskrankenpflegerinnen, die besonders daran leiden, dass sie ihre Kolleginnen ein ganzes Jahr lang nicht mehr gesehen haben, weil sie nicht mehr am gemeinsamen Stützpunkt sein durften. Gut funktionierende Teams sind aus diesen Gründen auseinandergebrochen. Das Zwischenmenschliche ist dabei sehr oft verloren gegangen. Auf unserer Station konnten wir das hingegen kultivieren. Wir wissen auch, dass wir das pflegen müssen, und das wollen wir auch weiterhin!

Mubri: Die Wichtigkeit des Zusammenhalts im Team war uns natürlich schon vorher bewusst, weil wir häufig Krisen haben. Die Krise gehört zu unserem Alltag. Jetzt kam durch Corona eben noch eine andere Krise mit einer anderen Dimension dazu, weil natürlich auch wir Angst um unsere Gesundheit hatten. Aber die Grundstruktur Krise und das Umgehen mit Krisen, das war uns nicht fremd.

Amschl-Strablegg: Ja! Wir haben ja fast ausschließlich PatientInnen, die sich aller Wahrscheinlichkeit nach in der schlimmsten Krise ihres Lebens befinden. Apropos Redebedarf: Wir haben uns auch regelmäßig online mit unseren Ehrenamtlichen getroffen. Denen war dies ein großes Anliegen, denn diese waren zu Beginn der Pandemie ja auch alle quasi zu Hause ,eingesperrt'. Ich glaube jedoch, dass die Kontakt-Angebote für Professionelle - und hier rede ich primär von anderen Stationen - nicht in diesem Ausmaß genutzt geworden sind wie bei unseren Ehrenamtlichen, weil die meisten Ärzte, Ärztinnen oder PflegerInnen es einfach nicht gewohnt sind, irgendwo hinzugehen und sich auszutauschen, wie es einem geht. Für uns hingegen ist das ganz normal. Es hat in unserem Haus auch das Angebot der Seelsorge und der Psychotherapie für alle gegeben. Diese Nutzung hat sich meines Wissens auch in Grenzen gehalten.

Mubri: Die Haltung ist oft vielmehr jene: „Man schafft das schon alleine!“ Amschl-Strablegg: Ja, das glaube ich auch. Ich hatte kürzlich folgende Rückmeldung aus der Intensivstation: „Ich bin Intensivschwester - ich muss das auch aushalten!“ Ich bin der Meinung, dass viele von uns mehr 
auf sich selber schauen müssen. Da geht es um die Achtsamkeit sich selber gegenüber. Das muss man aber in guten Zeiten trainieren!

Platzer: Jürgen Habermas hat kürzlich geschrieben: „So viel Wissen über unser Nichtwissen und über den Zwang, unter Unsicherheit handeln und leben zu müssen, gab es noch nie. "Wie kann man also in Zeiten großer Unsicherheiten als Führungskraft Sicherheit geben und Vorbild sein?

Mubri: Spontan fällt mir der Gedanke ein, dass dies nur dann gelingen kann, wenn man selbst authentisch unterwegs ist.

Amschl-Strablegg: Dem kann ich mich nur anschließen!

„Einsamkeit lässt Menschen auch sterben - das weiß man."

Platzer: Fanden Sie die Verhältnismäßigkeit der Schutzmaßnahmen als angemessen?

Mubri: Was unsere Einheit betrifft: ja. Ich habe es jedoch sehr traurig empfunden, von manchen Pflegeheimen zu hören, dass alte, einsame Menschen quasi ,weggesperrt ${ }^{6}$ wurden. Das ist eine Maßnahme, die sich für mich tatsächlich nicht mit den Menschenrechten vereinbaren lässt und die für mich inhuman und zutiefst würdelos ist. Die Maßnahmen, wie sie bei uns dann tatsächlich auch umgesetzt worden sind, finde ich hingegen vollkommen in Ordnung, und da habe ich auch von allen PatientInnen ein großes Verständnis wahrgenommen und keinen "Kampf' dagegen.

Amschl-Strablegg: Da bin ich zwar grundsätzlich auch deiner Meinung, aber ich möchte dazu noch folgendes anmerken: Das klingt hier fast so, als seien für manche unbefriedigende Situationen in den Pflegeheimen ausschließlich die Betreiber verantwortlich. Das ist aber nicht so. Ich habe diesbezüglich mit vielen Leuten gesprochen. Die meisten befanden sich tatsächlich in einer furchtbaren Misere. Hätte man die Menschen nicht so geschützt, und viele wären verstorben, dann hätte es auch einen Aufschrei gegeben. Als Pflegeheim hätte man es in dieser Situation wohl niemandem recht machen können. Andererseits bin ich auch bei dir, weil Einsamkeit lässt Menschen auch sterben - das weiß man.

Mubri: Das soll auch nicht als vereinfachte Schuldzuweisung gegenüber Pflegeheimen verstanden werden. Das hätten wir als Gesellschaft anders lösen müssen.

Amschl-Strablegg: Wie sagte Kierkegaard so schön: „Das Leben wird vorwärts gelebt, kann aber nur rückwärts verstanden werden.“ 
Platzer: Welche ethischen Fragen sind verstärkt bei der Begleitung Sterbender während der Corona-Zeit aufgetaucht?

Mubri: Ich würde sagen, dass die Frage nach der Würde und der Wichtigkeit der Beachtung der Einzigartigkeit eines jeden Menschen vermehrt zum Vorschein gekommen ist. Neben all diesen Maßnahmen geht es zunächst immer um den konkreten Tod eines Menschen im Kontext von vielleicht notwendigen Präventivmaßnahmen. Was nun aber ein würdevolles Sterben bedeutet, das kann immer nur die betroffene Person beurteilen. Da müssen wir hinhören, und jenes dann dieser Person so gut es geht ermöglichen. Ich möchte als Beispiel wieder die Seelsorge heranziehen: Ich würde mir in meinem Sterben unbedingt eine seelsorgliche Begleitung wünschen; und ich würde mir vom seelsorgenden Menschen den Mut wünschen, dass er auch dann mich begleitet, wenn ein geringes Risiko für ihn entsteht, an einer hoffentlich nicht schweren Erkrankung deswegen zu erkranken. Mut und Verantwortung, das brauchen wir in dieser Welt einfach. Ohne sich darauf zu verlassen oder auszureden, dass dafür irgendjemand anderes irgendwann zuständig sein könnte.

\section{„Eine frisch desinfizierte Hand kann ich immer jemandem reichen. Das ist einfach eine Sache des Hausverstandes"}

Platzer: Weil Sie gerade das Thema, Würde angesprochen haben: Welche Auswirkungen hatten die ganzen Maßnabmen und Veränderungen auf Ihre PatientInnen?

Amschl-Strablegg: Den Leuten fehlte vor allem unsere Mimik. Sie verstanden uns schlechter, weil sie ja nicht auf unsere Lippen blicken konnten. Deshalb haben wir - so lange es nur ging - auch nur Schutzschilder getragen. Aber sonst hat es meines Erachtens bei uns eigentlich keine großen Veränderungen für PatientInnen gegeben - auf den Corona-Stationen allerdings sehr wohl, denn dort waren die Sicherheitsvorkehrungen ja viel strenger.

Mubri: Was ich schon auch erlebt habe und immer noch erlebe, ist, dass die Menschen nicht mehr berührt werden - von der Pflege natürlich schon -, aber sonst ist Berührung jetzt eher tabuisiert worden. Da hat sich auch bei uns etwas verändert. Ich gehe normalerweise mit jedem Patienten in Berührung. Momentan allerdings nur dann, wenn ich glaube, dass es die jeweilige Situation unbedingt verlangt. 
Amschl-Strablegg: In der Pflege geht es natürlich nicht ohne Berührung. Was ich aber diesbezüglich oft nicht verstehe, ist, dass ganz viele Menschen einfach begonnen haben, ihren Hausverstand auszuschalten. Wenn ein Mensch schwer krank ist, gehen wir mit diesem natürlich auch weniger in Berührung. Wenn ich nun aber das Zimmer eines normalen Palliativpatienten betrete, dann werde ich mir natürlich immer meine Hände desinfizieren. Diese sind dann sauber, und ich bin nicht mehr ansteckend. Ohne jemanden jetzt zu irgendetwas ermutigen zu wollen: Eine frisch desinfizierte Hand kann ich immer jemandem reichen. Das ist einfach eine Sache des Hausverstandes. Und wenn jemand sterbend ist, dann bräuchte ich nicht einmal das zu machen, denn diese Person stirbt gerade; und es ist in so einer Situation egal, ob meine Hand desinfiziert ist oder nicht.

Mubri: Hier geht es wiederum darum, Verantwortung wahrzunehmen. Es stimmt schon: Hygienemaßnahmen sind wichtig, aber wenn man sich vor und nach den Berührungen desinfiziert: Was soll dann sein?

Platzer: Hat Ihres Erachtens also die Bedeutung einer körperlichen Berührung zugenommen?

Mubri: So wie Wasser in der Wüste, ja - voll und ganz.

Amschl-Strablegg: Aber nicht nur bei den PatientInnen, sondern auch bei uns MitarbeiterInnen! Sich einander spüren - zum Beispiel das Umarmen einer Kollegin - ist mitunter extrem wichtig! Das Vermeiden körperlicher Nähe ist aber jetzt zum Alltag geworden, und ich fürchte, dass dies auch so bleiben wird. Obwohl ich eigentlich keine Angst hatte, habe ich mir das körperliche Distanz-Halten ebenso extrem angewöhnt und verinnerlicht. Das ist eigentlich eigenartig.

Platzer: Gab es Situationen mit PatientInnen, die Ihnen besonders im Gedächtnis geblieben sind?

Muhri: Auf unserer Station gab es wie erwähnt nur einen einzigen Patienten, der positiv war, und als wir diesen dann im Sterbeprozesses auf die Corona-Station haben bringen müssen, hat das unser Palliativ-Herz bluten lassen, weil wir niemanden in einer Sterbephase woanders hingeben wollen. Aber es war zum Schutz der anderen einfach notwendig.

Amschl-Strablegg: Mir fällt auch eine besondere Situation mit einem Patienten ein. Wir hatten zu Beginn der Corona-Pandemie im VinziDorf-Hospiz einen Patienten mit einer terminalen Erkrankung. Diese war zwar bereits weit fortgeschritten, aber der Patient war noch in einer guten Allgemeinverfassung. Sein Wunsch war, dass er noch einmal seine Füße in den Sand an der Adria stecken möchte. Und wir beide - Dr. Muhri 
und ich - haben tatsächlich begonnen, diese Reise nach Italien zu planen. Wir wollten mit einem Malteser-Krankenwagen ans Meer fahren, um dem Patienten seinen letzten Wunsch zu ermöglichen. Zunächst waren die Grenzen noch geschlossen, doch im Juni gingen sie endlich wieder auf, und wir wollten dann die Reise starten. Nur leider ist der Patient dann einen Tag vorher verstorben. Aber diese Reise war und ist immer für uns präsent, obwohl sie gar nicht stattgefunden hat.

Platzer: Palliative Care umfasst ja auch die Begleitung und Betreuung der Angehörigen. Was hat sich diesbezüglich in den vergangenen Monaten verändert?

Muhri: In unserer Einheit hat sich für die Angehörigen prinzipiell nicht viel verändert. Sie mussten bzw. müssen sich lediglich immer testen lassen bzw. einen Test vorweisen, bevor sie zu uns kommen, und sie müssen auch eine Maske tragen. Dann können sie wie immer ganz normal zu Besuch kommen.

\section{Platzer: Hat sich dabei im Lauf der Zeit etwas in deren Verhalten verändert?}

Amschl-Strablegg: Ja! Am Anfang waren alle total froh und dankbar, dass sie ihre Angehörigen besuchen konnten. Jetzt ist wieder alles selbstverständlich. Wenn wir heute manche Angehörigen auf die Maskenpflicht im Zimmer aufmerksam machen, dann müssen wir uns sogar manchmal anpöbeln lassen. Hier merkt man auch eine große Müdigkeit, was die Maßnahmen betrifft.

„Familiengespräche werden mit oder obne Corona weiterhin auch online durchgeführt werden können"

Platzer: Wie haben Sie Ibre Kommunikationskanäle während der Pandemie angepasst?

Mubri: Wir haben zum Beispiel von den Maltesern sechs iPads übergeben bekommen, mit denen Angehörige dann auch visuell mit isolierten PatientInnen Kontakt aufnehmen konnten.

Amschl-Strablegg: Auf diesem Gebiet wird es sicherlich zu nachhaltigen Veränderungen kommen, vor allem für Angehörige mit weit entferntem Wohnsitz. Familiengespräche werden mit oder ohne Corona weiterhin auch online durchgeführt werden können.

Mubri: Ja, der Sohn, der in Amerika wohnt, darf am Familiengespräch teilnehmen. 
Platzer: Wie konnten An- und Zugehörige von Menschen, die im Sterben lagen oder bereits verstorben waren, Abschied nehmen? Was ist diesbezüglich anders geworden?

Mubri: Bei uns auf der Station war und ist es so wie immer. An Corona erkrankte Sterbende durften von Angehörigen bei uns im Haus auch besucht werden, und entsprechend konnte das leibliche Abschiednehmen im Krankenzimmer auch passieren.

Amschl-Strablegg: Auch wenn jemand plötzlich verstorben ist, hat es im Anschluss daran bei uns Verabschiedungen gegeben. Diese wurden oft von unserer Seelsorgerin gestaltet. Auch diesbezüglich hat die Seelsorge wiederum einen immensen Beitrag geleistet. Diese hat für so viel Menschlichkeit in unserem Haus gesorgt. Nicht, dass die anderen Berufe diese nicht zum Ausdruck bringen könnten oder konnten, aber die Umstände haben einfach vieles nicht zugelassen, was dann von der Seelsorge wiederum übernommen wurde.

Platzer: Weil Sie wiederum die Seelsorge ansprechen: Ist es auch von Seiten der anderen Berufsgruppen zu einer, von Ihnen immer wieder diagnostizierten ,Aufwertung der Seelsorge' gekommen?

Mubri: Ich glaube, in unserer Einheit ist es zu einer Aufwertung der Seelsorge von einem schon relativ hohen Niveau auf ein jetzt wirklich adäquates Niveau gekommen. Wie es auf den anderen Stationen aussieht, das weiß ich nicht.

Amschl-Strablegg: Andere Stationen kann ich auch nicht beurteilen. Das hängt aber meines Erachtens maßgeblich von den Leitenden der Stationen ab. Wenn der Seelsorge von der Stationsleitung Raum gegeben wird, dann bekommt jene auch automatisch einen gewissen Stellenwert. Das hat dann Vorbildwirkung, weil die MitarbeiterInnen orientieren sich ja an der Leitung - bewusst oder unbewusst.

„Obwohl ich geimpft bin, würde ich eine verpflichtende Impfung als Voraussetzung für die Ausübung meines Berufes nicht wollen"

Platzer: Kommen wir zu einem anderen Bereich: Inwiefern kommt es zu Ihnen zu Diskussionen über das Thema, Impfung des Gesundheitspersonals'?

Mubri: Ich persönlich stehe einem jeden körperlichen Eingriff, der verpflichtend ist, skeptisch gegenüber. Wenn von einem Impfstoff jedoch mit hoher Wahrscheinlichkeit eine gute Wirkung und kaum Nebenwir- 
kungen zu erwarten sind, dann gilt es aber auch, verantwortlich mit dieser Ressource umzugehen und diese anzunehmen, wenn dieser Impfstoff dir angeboten wird.

Platzer: Die österreichische Bioethikkommission spricht in diesem Kontext nicht von einer Impflicht für das Gesundheitspersonal, sondern sieht eine Impfung gegen COVID-19 vielmehr als eine, Berufsausübungserfordernis für die Gruppe der Pflege- und Gesundheitsberufe. Wie stehen Sie einer solchen Forderung gegenüber?

Amschl-Strablegg: Ich sehe solche Forderungen sehr kritisch! Obwohl ich geimpft bin, würde ich eine verpflichtende Impfung als Voraussetzung für die Ausübung meines Berufes nicht wollen. Auch ich habe einige Zeit gebraucht, bis ich von dieser Impfung überzeugt war. Ich versuchte mich diesbezüglich einfach aus seriösen, wissenschaftlichen Quellen zu informieren. Dazu noch eine Anmerkung: In meinem Team sind rund zwei Drittel geimpft. Die Entscheidung für oder gegen eine Impfung soll jedoch jeder und jede selbst fällen. Ich kann aber nicht nachvollziehen, wenn ein nicht geimpftes Teammitglied trotz eines Schnupfens zum Beispiel unbedingt zur Arbeit kommen will, nur weil es glaubt, unentbehrlich zu sein. So etwas finde ich unverantwortlich, denn erstens werden dadurch andere einer potenziellen Gefahr ausgesetzt und zweitens hätte eine Infizierung weitreichende Folgen auf der organisatorischen und bürokratischen Ebene - Stichwort verpflichtende Quarantäne, Contact-Tracing, Sicherung und Aufrechterhaltung des Betriebs usw.

Platzer: Was glauben Sie als Pflegefachfrau, warum sich bei uns in Österreich sehr viele Pflegerinnen und Pfleger bis jetzt nicht haben impfen lassen?

Amschl-Strablegg: Ehrlich gesagt halte ich dies großteils für eine Trotzreaktion. Am Anfang gab es den viel zitierten Applaus für die Pflege, dann kam die Diskussion über Entschädigungen, und letztlich gab es für uns nur eine Bagatelle, nämlich einen einzigen ,schäbigen' Urlaubstag! Ich wollte diesen sogar verschenken, so erzürnt war ich darüber - und ich habe ihn gar nicht verschenken dürfen! Und dann hat es plötzlich geheißen: „Wir [die Pflegekräfte] müssen als erstes geimpft werden." Da kann ich nur darauf antworten: „Wir müssen gar nichts!“ Ich habe mich dann zwar später impfen lassen, aber einige Kolleginnen lehnen eine Impfung vielleicht nach wie vor aus den von mir vermuteten Gründen ab.

Ich möchte gerade in diesem Kontext auf die unglaubliche Flexibilität unserer MitarbeiterInnen in der Pflege aufmerksam machen: Es gab z. B. eine Situation, wo plötzlich eine ganze Station ohne Personal dagestanden 
ist. Am Karfreitag hat mich unsere Pflegedirektorin zu Mitternacht angerufen und hat mich gefragt, ob ich für den nächsten Tag überhaupt noch jemanden zum Arbeiten habe. In solchen Situationen sind dann immer andere KollegInnen eingesprungen und das ist auch jetzt noch so. Da herrscht eine unglaubliche Flexibilität, da gibt es kein „nein“, und da gibt es auch kein Jammern!

Platzer: Worauf führen Sie diese Grundhaltung des Pflegepersonals eigentlich zurück?

Amschl-Strablegg: Ich glaube, dass Pflegende mit einem überdurchschnittlichen Pflichtbewusstsein ausgestattet sind. Es gehört zur Pflege quasi als Dogma dazu, dass man dann, wenn jemand ausfällt, sofort bereit ist, einzuspringen. Das ist ein ungeschriebenes Gesetz. Das hat aber auch seine Kehrseite: So werden Menschen sehr leicht, verheizt ${ }^{\star}$.

Platzer: Kommen wir noch einmal zum Thema ,Impfung zurück: Sind eigentlich die PatientInnen auf Ihrer Station geimpft worden?

Mubri: Im Setting der Palliativstation wird meistens nicht geimpft. RisikopatientInnen im Haus werden aber schon geimpft.

Amschl-Strablegg: Da wäre es in unserer Abteilung vernünftiger, man würde die Angehörigen impfen.

„Wozu erlauben wir jetzt den, assistierten Suizid', wenn wir jedes Leben schützen wollen?"

Platzer: Wir leben nun schon fast eineinhalb Jahre mit der Pandemie. Wie sind Sie persönlich mit den Herausforderungen der vergangenen Monate umgegangen?

Amschl-Strablegg: Ich habe ganz normal weitergearbeitet, mein Leben hat sich eigentlich wenig verändert. Ich arbeite gleich viel wie immer.

Platzer: Was hat sich in dieser Zeit als Ressource erwiesen?

Amschl-Strablegg: Da greife ich ebenso auf dieselben Ressourcen zurück wie sonst auch: Natur, Familie, Haustiere.

Mubri: In Berührung bleiben, so gut es geht. Zu deinen genannten möchte ich für mich noch Musik und vor allem die Religion ergänzen. Dass diese in manchen Facetten nicht gefeiert werden konnte, war belastend hat aber dafür neue Facetten entstehen lassen. 
Platzer: Abschließend noch eine Frage: Welche allgemeinen Lehren ziehen Sie eigentlich aus der Pandemie?

Amschl-Strablegg: Wozu erlauben wir jetzt den assistierten Suizid, wenn wir jedes Leben schützen wollen? Pandemie und assistierter Suizid - das geht einfach nicht zusammen, das ist doch ein Widerspruch!

Mubri: Ich möchte es etwas allgemeiner formulieren: Für mich ist die Erkenntnis durch Corona das Bewusstsein, dass wir Menschen hier auf der Erde nichts Angreifbares haben, das uns hundertprozentige Sicherheit bietet. All dieses Sicherheitskorsett, das wir uns in der Gesellschaft aufgebaut haben, ist immer nur relativ und bleibt relativ. Meine Erkenntnis daraus ist, dass wir versuchen sollten, in gegenseitiger Solidarität zu einer gewissen Demut zu gelangen, miteinander achtsam umzugehen und trotzdem immer diese Endlichkeit vor Augen zu haben.

Platzer: Vielen herzlichen Dank für das Gespräch. Ich wünsche Ihnen weiterhin alles Gute! 
